OAI-PMH: http://www.indteca.com/ojs/index.php/Revista Scientific/oai

Ensayo Original / Original Essay

\title{
La Educación Holística en el Nivel de Primaria Venezolana: Una Visión Resiliente de la Educación
}

Autor: César Enrique López Arrillaga Universidad Latinoamericana y el Caribe, ULAC

prof.cesarlopez@gmail.com

Caracas, Venezuela

\section{Resumen}

El presente ensayo tiene como finalidad abordar la educación holista en el subsistema de educación primaria desde las perspectivas de Simpson (2010), en su obra Resiliencia Sociocultural, así mismo los aportes de Sambrano (2010), en cuanto a la Resiliencia, con el objeto de realizar una aproximación teórica de la posible vinculación de la educación holística en el subsistema de educación primaria venezolana generando una aproximación en la visión resiliente de la educación en el marco de la educación holística, que impulse una práctica docente centrada en la formación integral de los estudiantes con un acercamiento pedagógico profundamente humano que genere la educación de calidad que requiere la educación actual.

Palabras clave: educación; docente de escuela primaria; escuela. 


\title{
Holistic Education in the Level of Venezuelan Primary: A Resilient Vision of Education
}

\begin{abstract}
The present essay aims to address holistic education in the subsystem of primary education from the perspectives of Simpson (2010), in his work Sociocultural Resilience, likewise the contributions of Sambrano (2010), in terms of resilience, in order to perform a theoretical approach to the possible linking of holistic education in the Venezuelan primary education subsystem, generating an approach in the resilient vision of education within the framework of holistic education, which promotes a teaching practice focused on the integral formation of students with a deeply human pedagogical approach that generates the quality education that current education requires.
\end{abstract}

Keywords: education; primary school teacher; school. 


\section{Introducción}

El subsistema de educación primaria debe vincular en su práctica educativa, los actores del hecho educativo promoviendo una relación natural generando aprendizajes esenciales en la vida de los estudiantes para su formación integral y en concordancia con sus intereses personales de cada uno como ser humano.

Así mismo, la educación primaria es un escenario ideal donde los estudiantes adquieren, construyen y comparten conocimientos mediante la interrelación de los demás actores del hecho educativa, entendiendo la escuela como un espacio integral de formación para todos sus integrantes.

Por otro lado, la resiliencia es la capacidad que poseen los seres humanos de adaptarse o vencer los obstáculos y dificultades en sus vidas, permite que a pesar de cualquier situación contraria en el desarrollo de sus actividades formativas los estudiantes adquieran herramientas para vencer los miedos, situaciones y problemas que diariamente experimentan en todos sus ámbitos de vida.

Por lo tanto, el presente ensayo se propone realizar una revisión teórica que fundamente la práctica holista en la escuela de educación primaria, que propicie en los estudiantes sus capacidades resilientes para vencer todas las barreras que existen en el sistema educativo en especial en el subsistema de educación primaria, causado por el momento histórico que atraviesa la humanidad producto de la globalización y la posmodernidad actual, en el cual el docente juega un papel protagónico como mediador y facilitador del proceso de enseñanza y aprendizaje.

\section{Desarrollo}

\subsection{La Resiliencia y sus fuentes}

La resiliencia es la capacidad que poseen los seres humanos para atravesar situaciones contrarias a las deseadas y traumáticas de estrés en la 
vida cotidiana, y como el individuo es capaz de vencerlas y superarlas naturalmente, y más allá seguir adelante convirtiéndolas en forma positiva para seguir con su vida normalmente.

Para Sambrano (2010a), la resiliencia se define como "la capacidad que tienen las personas para reaccionar satisfactoriamente ante las adversidades." (pág. 17). Por lo cual, es conveniente establecer que todos los seres humanos podemos desarrollar la capacidad de superar todos los obstáculos de la vida.

De acuerdo con el autor, los seres humanos en especial en su faceta de estudiantes durante la persecución de sus estudios de primaria, viven diversas situaciones que en la mayoría de los casos no pueden vencer, como por ejemplo: problemas económicos, acoso escolar y familias disfuncionales, dentro de este contexto el estudiante requiere de desarrollar habilidades que les permitan seguir adelante a pesar de las circunstancias adversas que viven o están expuestos.

Además, autor expone que "Todos los seres humanos estamos expuestos a situaciones adversas, al estrés, a la falta de amor, al maltrato. Algunas personas sucumben, evidenciando trastornos y desequilibrio; ostras fuerzas de sus flaquezas, asumen su potencialidad y superan la situación". (pág. 17).

Es por ello, que la vida de los estudiantes en el subsistema de educación primaria venezolana, experimenta un sinfín de situaciones adversas producto de la crisis social que vive el país actualmente, escasez de comida, inseguridad, violencia escolar, entre otros afectan la actuación en la escuela y su rendimiento escolar, afectando su éxito académico.

Es significativo señalar la importancia que posee la resiliencia en los entornos educativos, principalmente en la educación primaria en función de fortalecer los comportamientos humanos frente a momentos o situaciones de crisis, como lo establece, Sambrano (2010b):

La resiliencia es una manera de ver los comportamientos 
humanos desde una perspectiva multidisciplinaria que tiene como objetivo mejorar los procesos y resultados de los grupos humanos frente a las crisis, por su concepción holística que tiene su máxima posibilidad de valores y cultura de la sociedad. (pág. 20).

Por otra parte, Vanistendael (1994), citado por Sambrano (2010c):

La resiliencia distingue dos componentes: la resiliencia frente a la destrucción; es decir, la capacidad de proteger la propia integridad bajo la presión; por otra parte, más allá de la resiliencia, es la capacidad de forjar un comportamiento vital positivo pese a circunstancias difíciles. (pág. 20).

De acuerdo con el autor, la Resiliencia es una herramienta que permite la protección y el desarrollo de un comportamiento en los estudiantes, donde adquieren capacidad de una actitud positiva ante situaciones de estrés y superar las adversidades diarias en sus vidas.

Ahora bien, Sambrano (2010d), presenta algunas características que permiten identificar estudiantes resilientes, tales como:

- Congruencia entre lo que decimos y lo que hacemos.

- Formación docente.

- Educación para una acción reflexionada.

- Educación desde y para una realidad cambiante.

- Educación para la formación del ser integral.

- Motivación e interés.

- Enfoque transversal y transdisciplinario.

- Coherencia entre fines y medios.

En consecuencia, los estudiantes resilientes deben presentar ciertas y determinadas características que evidencien el desarrollo y apropiación de las fuentes de la resiliencia, que permitan la confrontación de adversidades diarias 
en los diversos contextos como en la familia, escuela o grupos de amigos, que alinea sus relaciones interpersonales en el interés de un ambiente de armonía.

\subsection{La Escuela como factor de práctica educativa holística}

Simpson (2010a), señala que la escuela: "tiene el objetivo de educar y sus integrantes toman ese rol concreto en la sociedad, diferenciados de otras instituciones" (pág. 9).

De acuerdo con el autor, la escuela representa un espacio diverso que debe ser orientado a educar a todos los integrantes con la finalidad de generar la integración de sus estudiantes a la sociedad actual, siendo el lugar ideal para forjar los ciudadanos que guiaran el futuro de las futuras generaciones.

Además, la escuela es el espacio propicio para promover estrategias y fortalecer la formación integral de los estudiantes con la finalidad de educar en valores para las generaciones de buenos ciudadanos capaces y eficaces para la sociedad que requiere el país, que responda a las necesidades colectivas de desarrollo integral.

Por otra parte, Simpson (2011a), señala que "la escuela junto con la familia, es la institución social que contribuye a conformar la identidad, en lo personal y en lo social" (pág. 9). De esta manera, la familia junto a la escuela son los espacios que pueden dar las herramientas necesarias para los estudiantes desarrollen y construyan su personalidad con ellos mismo y su entorno social.

Sin embargo, acompañado con la tarea institucional de la escuela, encontramos a la familia como una institución social en la cual se fundamentan los pilares de valores en la formación inicial y base de los estudiantes, es allí desde práctica diaria del hogar donde se da forma al ser social con todas sus características en lo personal y en lo colectivo. 
Por otra parte, Ehrensaf, E. y Tousignant, M. (2003), citados por Simpson (2011b), explica que:

El colegio es otra fuente básica de apoyo para el niño expuesto a sucesos o a un ambiente de vida de riesgo. El medio escolar es la segunda fuente de seguridad después del hogar y a veces, la única... es una de las instituciones cuya presencia es más constante en la vida del niño, y en los sistemas sociales modernos es la única de desarrollo principal después de la familia. (pág. 163).

Así mismo, la autora en su obra de la resiliencia sociocultural establece que el pronombre "Yo" se transforma en "Nosotros" que muestra la dimensión colectiva de esta capacidad. Así mismo, permite ubicar voluntades individuales a enfrentarlas en equipo de seres humanos, unidos con una determinación determinada. Como se puede observar en la gráfica $N$ ․․ 1, Fuentes de la Resiliencia Sociocultural.

En efecto, las fuentes de la resiliencia sociocultural se fundamentan en el quehacer colectivo, por que plantea una práctica colectiva, donde en sociedad se puede construir un nuevo orden social, un país orientado a satisfacer las necesidades colectivas por encima de las individuales.

Igualmente, se puede destacar que cada estudiante aporta su individualidad, en función de la corresponsabilidad y parte de una comunidad, institución, familia o grupo de trabajo orientados para el bien común y la satisfacción de las necesidades de formación colectivas centradas en el ser humano. 
Para lo cual, se presenta la Gráfica N. $\cong 1$, basada en las fuentes de la resiliencia sociocultural de Simpson (2010b), en una adaptación propia:

\section{Gráfica N. ำ 1 . Fuentes de la Resiliencia Sociocultural.}

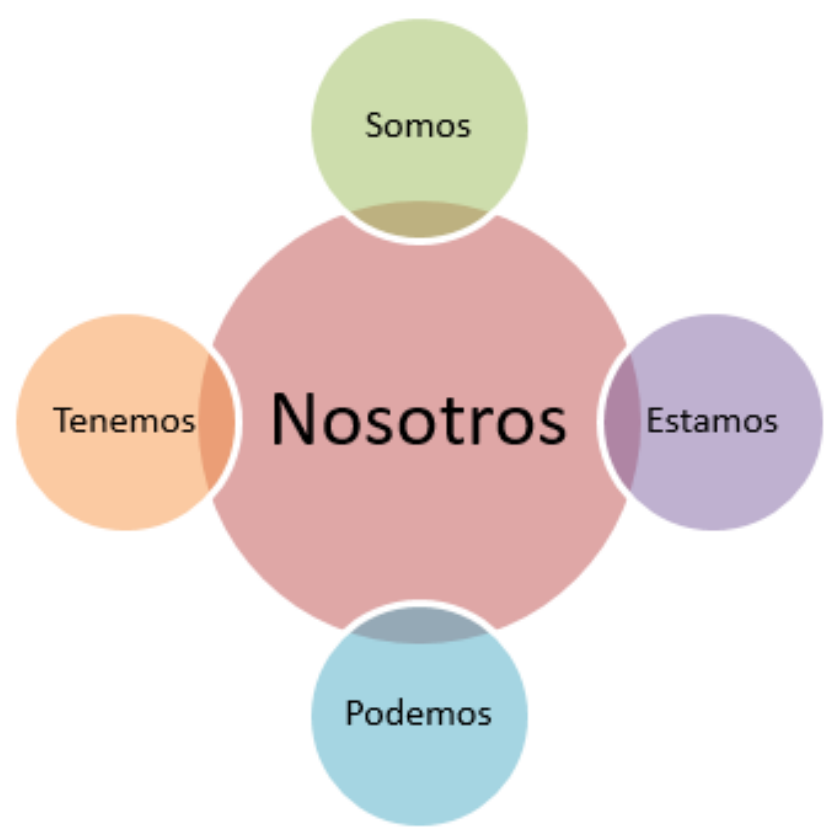

Fuente: Simpson (2010); adaptación propia del Autor (2017).

En la fuente de "Somos", plantea el respecto de los propios estudiantes y en el de los demás, permitiendo una felicidad colectiva cuando se realiza actos de buena voluntad para los demás y existen demostraciones de afectos mutuos consolidando los lazos de amistad y compañerismo.

A este respecto, la fuente de "Estamos", permite la disposición de los estudiantes de responsabilizarse de sus actos y las consecuencias que poseen el mismo, además ofrece la seguridad de la certeza que todo saldrá bien.

Además, en la fuente "Podemos", promueve la búsqueda de la forma de cómo resolver los problemas y reconocer la necesidad de encontrar a alguien que nos ayude cuando lo necesitamos, en consecuencia, prevé el 
control de sí mismo cuando tenemos ganas de hacer algo peligro o que no esté bien.

Por ello, en el tema de las fuentes de la resiliencia sociocultural se observa el "Tenemos", que promueve observa que se posee en el entorno con personas que se puede confiar y que quieren al ser humano incondicionalmente, además personas que nos muestran por medio de su conducta la manera correcta de proceder.

Cabe resaltar que las fuentes mencionadas al desarrollarlas en la escuela, permiten un clima escolar donde los estudiantes podrán desenvolver todas sus capacidades en armonía con sus iguales, aplicando sus capacidades de aprendizaje y relaciones interpersonales con la fortaleza interna y externa de afrontar cualquier situación de la vida.

\subsection{Educación holística como elemento la práctica educativa resiliente}

Para Simpson (2010c), establece que la Educación:

es un proceso social y humano por el cual un sujeto completa su desarrollo, ya que requiere adiestramiento para enfrentar la adversidad, compensando la información instintiva de la que carece y que a los animales les permite encontrar respuestas frente a las situaciones problemáticas que la realidad les impone. (pág. 18).

Igualmente, la autora indica que la escuela "es la continuadora de su tarea. Por eso, naturalmente, son los escenarios ideales para desarrollar la capacidad para enfrentar la adversidad, ósea, las fuentes de la resiliencia por excelencia" (pág. 18). De igual manera, se debe considerar la escuela como la institución encargada de perpetuar valores, generar pertenencia y cultura en los estudiantes que permitan una formación holística con capacidad resiliente para confrontar todas las situaciones difíciles de la vida cotidiana del ser humano. 
Por otro lado, Vanistendael (2006), citado por Simpson (2011c), indica que:

la resiliencia es una capacidad que se construye en la interacción entre el individuo y su entorno. Dicha capacidad se construye y se aumenta toda la vida, pero con altibajos y de formas concretas que puedan transformarse con el tiempo. (pág. 237).

De acuerdo con lo anterior, la escuela debe ser el entorno adecuado donde los estudiantes puedan fortalecer y desarrollar todas las capacidades, habilidades y destrezas en función de vencer todos los altibajos y conflictos que vivirá en su vida, para lograr un proceso de enseñanza aprendizaje de acuerdo con sus intereses de formación en un ambiente saludable y cordial.

Por tanto, permitiendo la inclusión de la práctica resiliente de los actores del sistema educativo en un proceso educativo más humano y en concordancia con las necesidades y realidad de la sociedad actual en globalización y posmodernidad que existe en el mundo.

Para lo cual, se puede observar en la gráfica $N$ ․․ 2, que nos permite identificar los elementos de una educación holística que promueve la interacción del hecho educativo planteado desde una perspectiva resiliente.

Grafica N.․ 2. La educación holista desde una perspectiva resiliente.

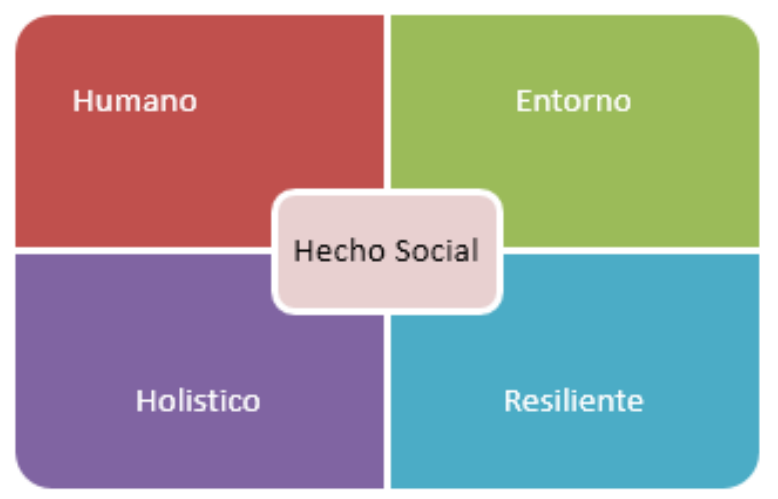

Fuente: Simpson (2011), adaptación propia del Autor (2017). 
En concordancia, la educación debe ser considerada como un hecho social donde el ser humano interactúa con su entorno colectivo, en el marco de la práctica holística partiendo de sus intereses de formación de sus habilidades y destrezas, en función de fortalecer las capacidades resilientes que le permitan confrontar todas las posibles adversidades de la cotidianidad en la sociedad actual.

Para lo cual, la educación debe promover la práctica formativa y de generación de aprendizaje en el ser humano como elemento fundamental del proceso de enseñanza, con métodos y estrategias holísticas que permitan la aprehensión de capacidades resilientes en la construcción de seres humanos con habilidades, destrezas y capacidades para la vida.

Por lo tanto, para que los docentes generen una práctica educativa resiliente, se debe propiciar en cada uno de ellos, un pensamiento flexible en su relación al proceso de enseñanza-aprendizaje, en cuanto educan a seres humanos con su complejidad, de acuerdo con (Riso, 2007, pág. 15), "La fuerza del pensamiento flexible radica en que, a pesar de la resistencia y los obstáculos, nos permiten inventarnos a nosotros mismos y fluir con la vida sin lastimar ni lastimarse".

\section{Conclusiones}

La Resiliencia como capacidad de los seres humanos de confrontar de manera positiva las adversidades y crisis que ocurren en su vida personal y académica, por ello los estudiantes deben fortalecer su habilidad de vencer todas las limitaciones con el propósito de alcanzar el éxito en su formación.

Dentro de esta perspectiva, la Educación es considerada como el proceso donde los estudiantes se permitan construir e intercambiar sus saberes y conocimientos de forma holística, centrada en sus intereses y necesidades de formación, llena de los valores humanistas más preciados en función de moldear los ciudadanos que requiere la sociedad actual. 
En esta misma perspectiva, la escuela y la familia como las principales instituciones sociales, juegan un papel importante en la formación de los ciudadanos que requerimos para confrontar los retos y desafíos del presente siglo y el momento histórico que atraviesa el mundo educativo.

Es importante destacar que, la familia juega un papel importante en la educación de las futuras generaciones aunado al rol que juega los docentes en la escuela primaria, el cual debe ser mediador y facilitador de los procesos de enseñanza-aprendizaje centrado en los seres humanos, sensible, cooperativo, y en particular el hecho educativo mediado por el amor a los demás.

Por otra parte, se debe crear en la praxis docente una visión holística para atender los desafíos que nos prepara los estudiantes del siglo XXI, una comunidad educativa globalizada con tendencia tecnológica, con un cumulo de intereses diversos, que le permite el acceso inmediato a la información y al conocimiento.

Además, el docente está invitado a vivir y expandir el amor en todos los espacios de la escuela, en especial su relación con los estudiantes. (Sepúlveda, 2008, pág. 173), "El amor es el alimento del alma”, por ende, nuestros estudiantes son instrumentos que la vida pone en el camino de los docentes para dar y recibir amor, como practica sublime de la educación.

\section{Referencias}

Riso, W. (2007). El poder del pensamiento flexible. De una mente rígida a una mente libre y abierta al cambio. Bogotá, Colombia: Grupo Editorial Norma, págs. 26-78.

Sambrano, J. (2010a,b,c,d). Resiliencia, transformación positiva de la adversidad. Caracas, Venezuela: Editorial Alfa. 1er reimpresión, págs. 15-98.

Sepúlveda, M. (2008). El buen vivir. Venezuela: Editorial Ediciones B, 
Venezuela S.A., págs. 59-78.

Simpson, M. (2011a,b,c). Resiliencia en el aula, un camino posible. Buenos Aires, Argentina: Editorial Bonum, Cuarta Edición, págs. 19-43.

Simpson, M. (2010a,b,c). Resiliencia sociocultural, Del "Yo puedo" al "Nosotros podemos". Buenos Aires, Argentina: Editorial Bonum, págs. 15-175.

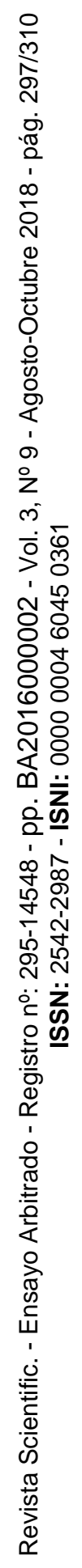

309 


\section{César Enrique López Arrillaga}

e-mail: prof.cesarlopez@gmail.com

Nacido en La Guaira, Venezuela. Licenciado en

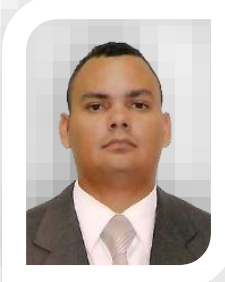

Educación, Mención: Desarrollo Cultural de la Universidad Nacional Experimental Simón Rodríguez (UNESR), Magister Scientiarum en Educación Superior de la Universidad Nacional Experimental de la Fuerza Armada (UNEFA), Doctorante del programa Ciencias de la Educación de la Universidad Latinoamericana y del Caribe (ULAC), docente de educación primaria de la Unidad Educativa Nacional Bolivariana Guaicaipuro.

El contenido de este manuscrito se difunde bajo una Licencia de Creative Commons Reconocimiento- 